SLAC-PUB-9020

BABAR-PROC-01/74

hep-ex/0000

October, 2001

\title{
Hadronic B decays to open charm at the BABAR Experiment
}

\author{
Jochen R. Schieck \\ University of Maryland \\ Department of Physics \\ College Park, MD 20742 \\ E-mail : schieck@slac.stanford.edu \\ (for the BABAR Collaboration)
}

\begin{abstract}
Using about $23 \mathrm{M} B \bar{B}$ events collected in 1999-2000 with the BABAR detector, we report on the decays $B \rightarrow D^{(*)} \bar{D}^{(*)} K$ and $B^{0} \rightarrow D^{*+} D^{*-}$. The branching fractions of the low background decay modes $B^{0} \rightarrow D^{*-} D^{(*) 0} K^{+}$are determined to be $\mathcal{B}\left(B^{0} \rightarrow D^{*-} D^{0} K^{+}\right)=(2.8 \pm 0.7 \pm 0.5) \times 10^{-3}$ and $\mathcal{B}\left(B^{0} \rightarrow D^{*-} D^{* 0} K^{+}\right)=(6.8 \pm 1.7 \pm 1.7) \times 10^{-3}$, where the first error quoted is statistical and the second systematic. Observation of a significant number of candidates in the color-suppressed decay mode $B^{+} \rightarrow D^{*+} D^{*-} K^{+}$is reported with a branching fraction $\mathcal{B}\left(B^{+} \rightarrow D^{*+} D^{*-} K^{+}\right)=$ $(3.4 \pm 1.6 \pm 0.9) \times 10^{-3}$. Decays of the type $B \rightarrow D^{(*)} \bar{D}^{(*)}$ can be used to provide a measurement of the parameter $\sin 2 \beta$ of the Unitarity Triangle. For this decay mode we measure a branching fraction of $\mathcal{B}\left(B^{0} \rightarrow D^{*+} D^{*-}\right)=(8.0 \pm 1.6 \pm 1.2) \times 10^{-4}$. All results presented here are preliminary.

Contributed to the Proceedings of the International Europhysics Conference on High Energy Physics, 7/12/2001-7/18/2001, Budapest, Hungary
\end{abstract}

Stanford Linear Accelerator Center, Stanford University, Stanford, CA 94309

Work supported in part by Department of Energy contract DE-AC03-76SF00515. 


\section{Introduction}

Decays of $B$ mesons that include a charmed and an anti-charmed meson are expected to occur through the $b$ to $c$ quark transitions $\bar{b} \rightarrow \bar{c} W^{+}$, where the $W^{+}$materializes as a $c \bar{s}$ pair. These transitions are responsible for most of the $D_{s}$ production in $B$ decays. $D_{s}$ production has been thoroughly studied in experiments running at the $\Upsilon(4 S)$ resonance [1], 2, 3]. The inclusive rate for $D_{s}$ production in $B$ decays was recently measured by $B A B A R$, where a preliminary branching fraction $\mathcal{B}\left(B \rightarrow D_{s} X\right)=(10.93 \pm 0.19 \pm 0.58 \pm 2.73) \times 10^{-2}$, the third error being due to the $D_{s}^{+} \rightarrow \Phi \pi^{+}$branching fraction uncertainty, is reported 洶.

Until 1994, it was believed that the $c \bar{s}$ quarks would hadronize dominantly as $D_{s}^{+(*)}$ mesons. Therefore, the branching fraction $b \rightarrow c \bar{c} s$ was computed from the inclusive $B \rightarrow D_{s} X, B \rightarrow$ $(c \bar{c}) X$ and $B \rightarrow \Xi_{c} X$ branching fractions, leading to $\mathcal{B}(b \rightarrow c \bar{c} s)=15.8 \pm 2.8 \%$ [5]. Theoretical calculations are unable to simultaneously describe this low branching fraction and the semileptonic branching fraction of the $B$ meson [6]. It has been conjectured [7] that $\mathcal{B}(b \rightarrow c \bar{c} s)$ is in fact larger and that decays $B \rightarrow D \bar{D} K(X)$ (where $\mathrm{D}$ can be either a $D^{0}$ or a $D^{ \pm}$) could contribute significantly. This might also include possible decays to orbitally-excited $D_{s}$ mesons, $B \rightarrow \bar{D}^{(*)} D_{s}^{* *}$, followed by $D_{s}^{* *} \rightarrow D^{(*)} K$.

In $B A B A R$, the high statistics available allow comprehensive investigations to be made of the $b \rightarrow c \bar{c} s$ transitions. In the analysis described in this paper, we present evidence for the decays $B \rightarrow$ $D^{(*)} \bar{D}^{(*)} K_{S}^{0}$ and $B \rightarrow D^{(*)} \bar{D}^{(*)} K$, using events in which both $D^{\prime}$ s are completely reconstructed. After describing the data sample and the event selection, we show the $D^{(*)} \bar{D}^{(*)} K$ signals for the sum of all $B$ submodes. The branching fractions for some of the cleanest modes, such as $B^{0} \rightarrow$ $D^{*-} D^{(*) 0} K^{+}$円, are computed. Observation of several candidates in the color-suppressed decay mode $B^{+} \rightarrow D^{*-} D^{*+} K^{+}$is also reported.

One of the most important goals of the BABAR experiment is to precisely measure the angles of the Unitarity Triangle. While the decay $B^{0} \rightarrow J / \psi K_{S}^{0}$ can be used to measure $\sin 2 \beta$, the Standard Model predicts that the time-dependent $C P$ violating asymmetries in the decays $B^{0} \rightarrow D^{(*)+} D^{(*)-}$ can also be used to measure the same quantity. An independent measurement of $\sin 2 \beta$ in these modes would therefore provide a consistency test of $C P$-violation in the Standard Model. The vector-vector decay $B^{0} \rightarrow D^{*+} D^{*-}$ is not, however, a pure $C P$ eigenstate. A sizeable dilution of the measured asymmetry may be produced by a non-negligible $P$-wave $C P$-odd component. The dilution can, in principle, be completely removed by a time-dependent angular analysis of the decay products [8].

A detailed description of the BABAR detector can be found elsewhere [9].

\section{Analysis}

Since the $B$ mesons are produced via $e^{+} e^{-} \rightarrow \Upsilon(4 \mathrm{~S}) \rightarrow B \bar{B}$, the energy of the $B$ in the $\Upsilon(4 S)$ frame is given by the beam energy $E_{\text {beam }}^{*}$, which is measured much more precisely than the energy of the $B$ candidate. Therefore, to isolate the $B$ meson signal, we use two kinematic variables: $\Delta E$, the difference between the reconstructed energy of the $B$ candidate and the beam energy in the center of mass frame, and $m_{\mathrm{ES}}$, the beam energy substituted mass, defined as $m_{\mathrm{ES}}=\sqrt{E_{\text {beam }}^{* 2}-p_{B}^{* 2}}$ where $p_{B}^{*}$ is the momentum of the reconstructed $B$ in the $\Upsilon(4 S)$ frame. Signal events will have $\Delta E$ close to 0 and $m_{\mathrm{ES}}$ close to the $B$ meson mass, $5.729 \mathrm{GeV} / c^{2}$.

\footnotetext{
${ }^{1}$ Charge-conjugate reactions are implied throughout this note.
} 


\section{$2.1 \quad B \rightarrow D^{(*)} \bar{D}^{(*)} K$ decays}

The $B^{0}$ and $B^{+}$mesons are reconstructed in a sample of multihadron events for all possible $D \bar{D} K$ modes, namely $B^{0} \rightarrow D^{(*)-} D^{(*) 0} K^{+}, D^{(*)-} D^{(*)+} K^{0}, \bar{D}^{(*) 0} D^{(*) 0} K^{0}$ and $B^{+} \rightarrow \bar{D}^{(*) 0} D^{(*)+} K^{0}$, $\bar{D}^{(*) 0} D^{(*) 0} K^{+}, \bar{D}^{(*)+} D^{(*)-} K^{+}$. The $D^{0}$ and $D^{+}$mesons are reconstructed in the modes $D^{0} \rightarrow$ $K^{-} \pi^{+}, K^{-} \pi^{+} \pi^{0}, K^{-} \pi^{+} \pi^{-} \pi^{+}$and $D^{+} \rightarrow K^{-} \pi^{+} \pi^{+}$. Charged kaon identification, with information from the Cherenkov angle in the DIRC and from $\mathrm{d} E / \mathrm{d} x$ measurements in the drift chamber and in the vertex detector, is required for most $D$ decay modes, as well as for the $K^{ \pm}$from $B^{\prime}$ 's.

$D^{*}$ candidates are reconstructed in the modes $D^{*+} \rightarrow D^{0} \pi^{+}, D^{* 0} \rightarrow D^{0} \pi^{0}$ and $D^{* 0} \rightarrow D^{0} \gamma$, by combining a $D^{0}$ candidate with a $\pi^{-}, \pi^{0}$, or photon. Partial reconstruction of $D^{* 0}$ 's (no $\pi^{0}$ or $\gamma$ reconstruction) is also used in the $B^{0} \rightarrow D^{*-} D^{* 0} K^{+}$mode, as explained below. $B$ candidates are reconstructed from the $D^{(*)}, \bar{D}^{(*)}$ and $K$ candidates. A mass constraint is applied to all the intermediate particles $\left(D^{0}, D^{-}, K_{S}^{0}\right)$. When several candidates are selected per event in a specific $B$ submode (e.g. $B^{+} \rightarrow D^{0} \bar{D}^{0} K^{+}$), a $\chi^{2}$ value, taking into account the difference between the measured and the PDG values of the $D$ masses and of the $\Delta M$ (for $D^{*}$ 's) is constructed and only the candidate with the lowest $\chi^{2}$ value is kept for the given submode.

\subsubsection{Evidence for signal in the sum of all $B$ submodes}

We present here the distributions obtained by summing all possible $B \rightarrow D^{(*)} D^{(*)} K$ decay channels, for neutral and charged $B$ decays respectively. Since multiple candidates are removed only submode by submode the same event can appear several times in distributions obtained by summing over all modes. An event will appear in the peak near $0 \mathrm{MeV}$ when reconstructed correctly, in the peak around $-160 \mathrm{MeV}$ if it is a $D^{*} D K\left(D^{*} D^{*} K\right)$ decay reconstructed as $D D K\left(D^{*} D K\right)$, and near the peak around $+160 \mathrm{MeV}$ if it is a $D D K\left(D^{*} D K\right)$ decay reconstructed as $D^{*} D K\left(D^{*} D^{*} K\right)$. About $120 \mathrm{~B}^{0}$ 's and $180 \mathrm{~B}^{ \pm}$decays have been reconstructed. The $m_{\mathrm{ES}}$ distributions (Figs. 1 and 2) contain only events with $|\Delta E|<24 \mathrm{MeV}$. The $m_{\mathrm{ES}}$ spectrum of $B^{0}$ and $B^{ \pm}$events can be fitted by the sum of a background shape and a Gaussian function used to extract the number of signal events. The background is empirically described by the ARGUS function [10].

\subsubsection{Measurement of exclusive branching fractions}

In this section, we present measurements of branching fractions for the three decay channels $B^{0} \rightarrow$ $D^{*-} D^{0} K^{+}, B^{0} \rightarrow D^{*-} D^{* 0} K^{+}$and $B^{+} \rightarrow D^{*-} D^{*+} K^{+}$. Several candidates are also observed in the $C P$ eigenstate $B^{0} \rightarrow D^{(*)-} D^{(*)+} K_{S}^{0}$ but without extracting branching fractions.

The selection efficiencies for each mode were obtained from detailed Monte Carlo simulation [11]. Typical efficiencies range from $10 \%$, for $B^{0} \rightarrow D^{*-} D^{0} K^{+}$with both $D^{0}$ 's decaying to $K \pi$, to less than $1 \%$, for $B^{+} \rightarrow D^{*-} D^{*+} K^{+}$with $D^{0}$ 's decaying to $K \pi \pi^{0}$ or $K 3 \pi$. Systematic errors account for the uncertainties on tracking and $\pi^{0}$ reconstruction efficiencies, $K$ identification efficiency, $D$ and $B$ vertexing requirements, efficiency of the requirement on $\Delta E$ used to define the signal box, efficiency of the $D$ mass requirement, uncertainty on the background shape, uncertainties on the $D$ and $D^{*}$ branching fractions, uncertainties on the selection efficiencies arising from Monte Carlo statistics, and uncertainty on the number of produced $B \bar{B}$ events in the data sample. For the decay $B^{+} \rightarrow D^{*-} D^{*+} K^{+}$a fit is performed with the sum of a Gaussian function for the signal and an ARGUS function for the background. The number of signal events is $8.2 \pm 3.5$ and the number of background events given by the ARGUS function is 1.7. The probability that the signal arises from 


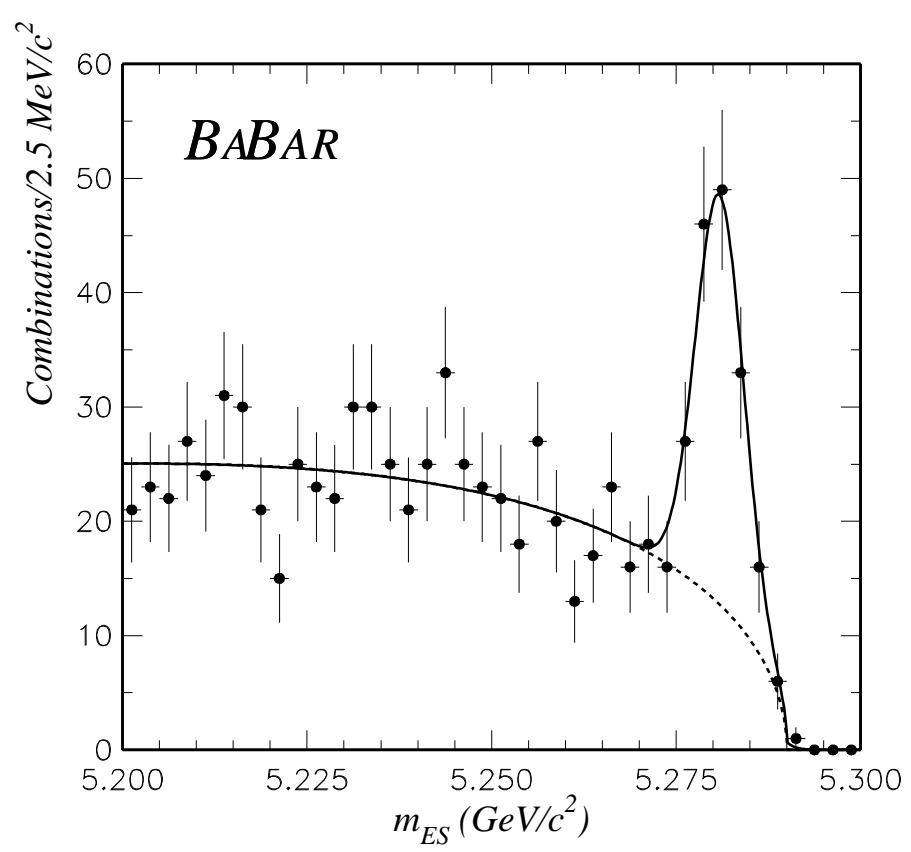

Figure 1: $m_{\mathrm{ES}}$ distribution for the sum of all neutral modes

a background fluctuation is $1.4 \times 10^{-5}(>5 \sigma)$. The corresponding preliminary branching fraction is measured to be $\mathcal{B}\left(B^{+} \rightarrow D^{*-} D^{*+} K^{+}\right)=(3.4 \pm 1.6 \pm 0.9) \times 10^{-3}$

In the analysis of the decay $B^{0} \rightarrow D^{*-} D^{(*) 0} K^{+}$we require that either the $D^{0}$ or the $\bar{D}^{0}$ decays to $K \pi$ and we do not explicitly reconstruct the $\pi^{0}$ or the photon from $D^{* 0} \rightarrow D^{0} \pi^{0}$ or $D^{0} \gamma$. Events containing $B^{0} \rightarrow D^{*-} D^{0} K^{+}$decays are selected by requiring $|\Delta E|<25 \mathrm{MeV}$. The number of signal events is found to be $29.6 \pm 7.2$. After correcting for the selection efficiencies and for the intermediate $D^{0}$ and $D^{*+}$ branching fractions [12], the preliminary branching fraction for $B^{0} \rightarrow D^{*-} D^{0} K^{+}$is found to be $\mathcal{B}\left(B^{0} \rightarrow D^{*-} D^{0} K^{+}\right)=(2.8 \pm 0.7 \pm 0.5) \times 10^{-3}$. Events containing $B^{0} \rightarrow D^{*-} D^{* 0} K^{+}$ decays are selected by requiring $|\Delta E+154|<60 \mathrm{MeV}$. The average position and width of $\Delta E$ for $B^{0} \rightarrow D^{*-} D^{* 0} K^{+}$is found to be in good agreement with expectations from $B^{0} \rightarrow D^{*-} D^{* 0} K^{+}$ signal Monte Carlo studies. The number of signal events found is $80.2 \pm 15.3$. To extract the $B^{0} \rightarrow D^{*-} D^{* 0} K^{+}$branching fraction, the contamination from decays $B^{+} \rightarrow D^{*-} D^{*+} K^{+}$, where the $\pi^{+}$from the $D^{*+}$ is not reconstructed, needs to be subtracted. This contribution has been estimated by performing the $B^{0} \rightarrow D^{*-} D^{* 0} K^{+}$analysis on $B^{+} \rightarrow D^{*-} D^{*+} K^{+}$signal Monte Carlo, assuming the $B^{+} \rightarrow D^{*-} D^{*+} K^{+}$branching fraction determined above. The $B^{+} \rightarrow D^{*-} D^{*+} K^{+}$ background contribution is estimated to be $20.6 \pm 9.7$ events. After subtracting this contribution, the preliminary $B^{0} \rightarrow D^{*-} D^{* 0} K^{+}$branching fraction is determined to be: $\mathcal{B}\left(B^{0} \rightarrow D^{*-} D^{* 0} K^{+}\right)=$ $(6.8 \pm 1.7 \pm 1.7) \times 10^{-3}$.

As pointed out in [13], the channel $B^{0} \rightarrow D^{*+} D^{*-} K_{S}^{0}$ is a $C P$ eigenstate that could be used for $\sin 2 \beta$ measurements. For decay channels $B^{0} \rightarrow D^{(*)-} D^{(*)+} K_{S}^{0}$ involving $D^{0}$ 's, at least one decay $D^{0} \rightarrow K \pi$ was required. The fitted number of signal events is $10.1 \pm 3.7$ with an estimated 


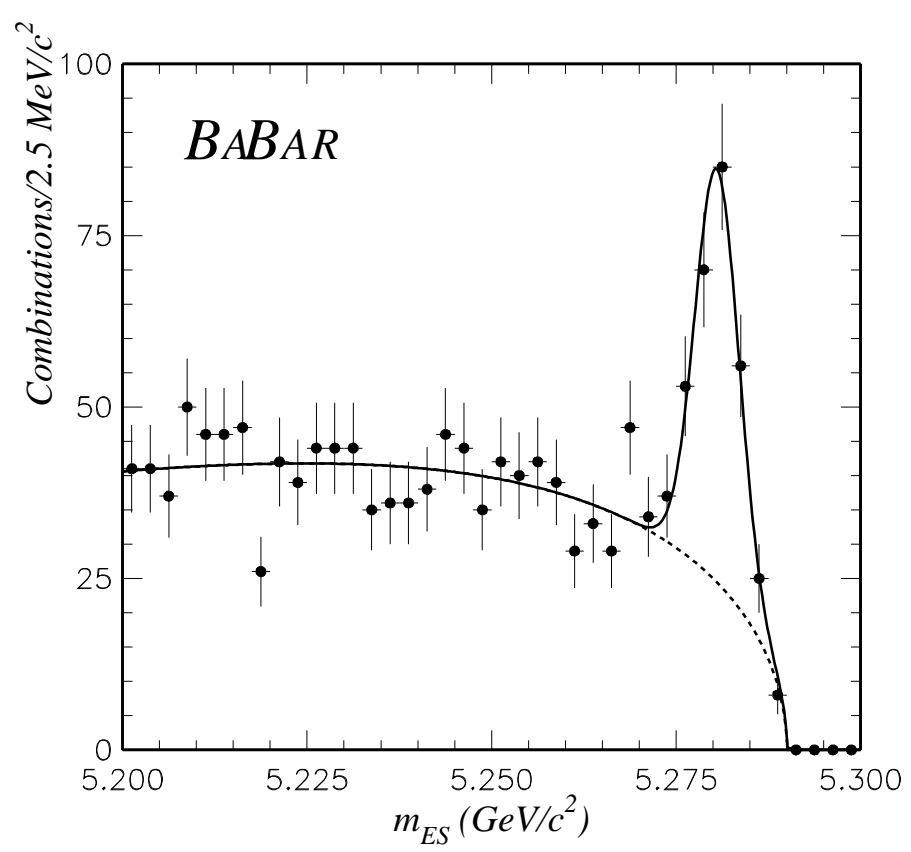

Figure 2: $m_{\mathrm{ES}}$ distribution for the sum of charged neutral modes

background of 3.4 events. The probability that the signal is a fluctuation of the background is $1.4 \times 10^{-5}(>5 \sigma)$. Most of the signal is due to the channels $B^{0} \rightarrow D^{*+} D^{-} K_{S}^{0}(4.7 \pm 2.2$ events with a background of 1 event) and $B^{0} \rightarrow D^{*+} D^{*-} K_{S}^{0}(4.8 \pm 2.2$ events with a background of 0.3 event).

\section{$2.2 \quad B^{0} \rightarrow D^{*+} D^{*-}$ decay}

$B^{0}$ mesons are exclusively reconstructed by combining two charged $D^{*}$ candidates reconstructed in a number of $D^{*}$ and $D$ decay modes. The decay modes of the $D^{0}$ and $D^{+}$used in this analysis were selected by an optimization of $S^{2} /(S+B)$ based on Monte Carlo simulations, where $S$ is the expected number of signal events and $B$ is the expected number of background events. $D^{0}$ and $D^{+}$candidates are reconstructed in the modes $D^{0} \rightarrow K^{-} \pi^{+}, D^{0} \rightarrow K^{-} \pi^{+} \pi^{0}, D^{0} \rightarrow K^{-} \pi^{+} \pi^{+} \pi^{-}$, $D^{0} \rightarrow K_{S}^{0} \pi^{+} \pi^{-}, D^{+} \rightarrow K^{-} \pi^{+} \pi^{+}, D^{+} \rightarrow K_{S}^{0} \pi^{+}$and $D^{+} \rightarrow K^{-} K^{+} \pi^{+}$. The $D^{*+}$ mesons are reconstructed in their decays $D^{*+} \rightarrow D^{0} \pi^{+}$and $D^{*+} \rightarrow D^{+} \pi^{0}$. We include for this analysis the decay combinations $D^{*+} D^{*-}$ decaying to $\left(D^{0} \pi^{+}, \bar{D}^{0} \pi^{-}\right)$or $\left(D^{0} \pi^{+}, D^{-} \pi^{0}\right)$, but not $\left(D^{+}\right.$ $\pi^{0}, D^{-} \pi^{0}$ ) due to the smaller branching fraction and larger expected backgrounds. $D^{0}$ and $D^{+}$ candidates are subjected to a mass-constraint fit and then combined with soft pion candidates. Charged kaon candidates are required to be inconsistent with the pion hypothesis, as inferred from the Cherenkov ring measured by the DIRC and the $\mathrm{d} E / \mathrm{d} x$ as measured by the SVT and DCH. To select $B^{0}$ candidates with well reconstructed $D^{*}$ and $D$ mesons, we construct a $\chi^{2}$ that includes all PDG and measured $D^{*}$ and $D$ masses. In events with more than one $B^{0}$ candidate, we chose the 
candidate with the lowest value of $\chi^{2}$.

The signal region in the $\Delta E$ vs. $m_{\mathrm{ES}}$ plane is defined to be $|\Delta E|<25 \mathrm{MeV}$ and $5.273<$ $m_{\mathrm{ES}}<5.285 \mathrm{GeV} / c^{2}$. The width of this region corresponds to approximately $\pm 2.5 \sigma$ in both $\Delta E$ and $m_{\mathrm{ES}}$. In addition only events with a $\chi^{2}<20$ are selected. To estimate the contribution from background in the signal region, we define a sideband in the $\Delta E$ vs. $m_{\mathrm{ES}}$ plane as $|\Delta E|<200 \mathrm{MeV}$, $5.20<m_{\mathrm{ES}}<5.26 \mathrm{GeV} / c^{2}$ and $50<|\Delta E|<200 \mathrm{MeV}, 5.26<m_{\mathrm{ES}}<5.29 \mathrm{GeV} / c^{2}$. We parameterize the shape of the background in the $\Delta E v s . m_{\mathrm{ES}}$ plane as the product of an ARGUS function in $m_{\mathrm{ES}}$ and a first-order polynomial in $\Delta E$. Based on this parameterization we estimate that the ratio of the number of background events in the signal region to the number in the sideband region is $(1.72 \pm 0.10) \times 10^{-2}$. Figure 3 shows the events in the $\Delta E$ vs. $m_{\mathrm{ES}}$ plane after all selection criteria have been applied. The small box in the figure indicates the signal region defined above, and the sideband is the entire plane excluding the region bounded by the larger box outside the signal region. There are a total of 38 events located in the signal region, with 363 events in the sideband region. The latter, together with the effective ratio of areas of the signal region to the sideband region, implies an expected number of background events in the signal region of $6.24 \pm 0.33 \pm 0.36$. The quoted systematic uncertainty comes from the background shape variation discussed previously. Figure 4 shows a projection of the data on to the $m_{\mathrm{ES}}$ axis after requiring $|\Delta E|<25 \mathrm{MeV}$.

We use a detailed Monte Carlo simulation of the BABAR detector to determine the efficiency for reconstructing the signal [11]. This, together with the total number of $B \bar{B}$ pairs produced during data collection, allows us to determine a preliminary branching fraction for $B^{0} \rightarrow D^{*+} D^{*-}$ to be $\mathcal{B}\left(B^{0} \rightarrow D^{*+} D^{*-}\right)=(8.0 \pm 1.6 \pm 1.2) \times 10^{-4}$.

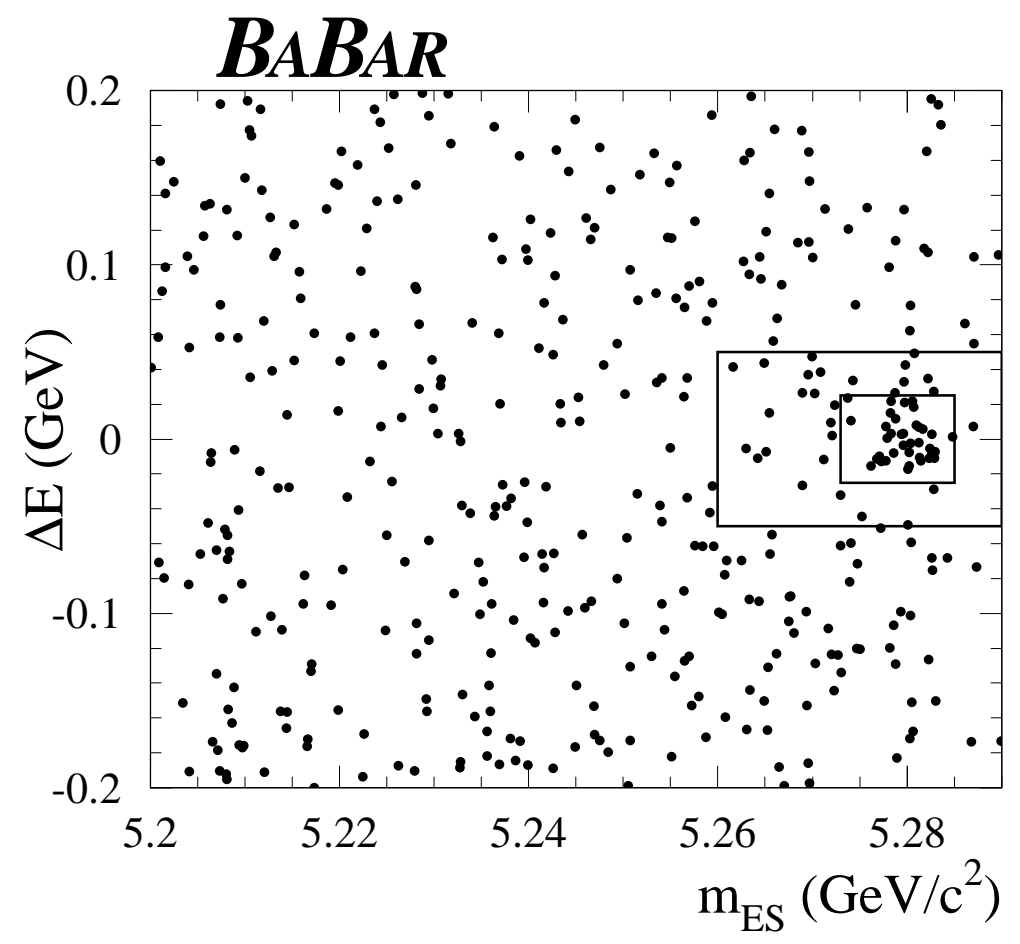

Figure 3: Distribution of events in the $\Delta E$ vs. $m_{\mathrm{ES}}$ plane. The small box indicates the signal region, while the sideband region is everything outside the larger box. 


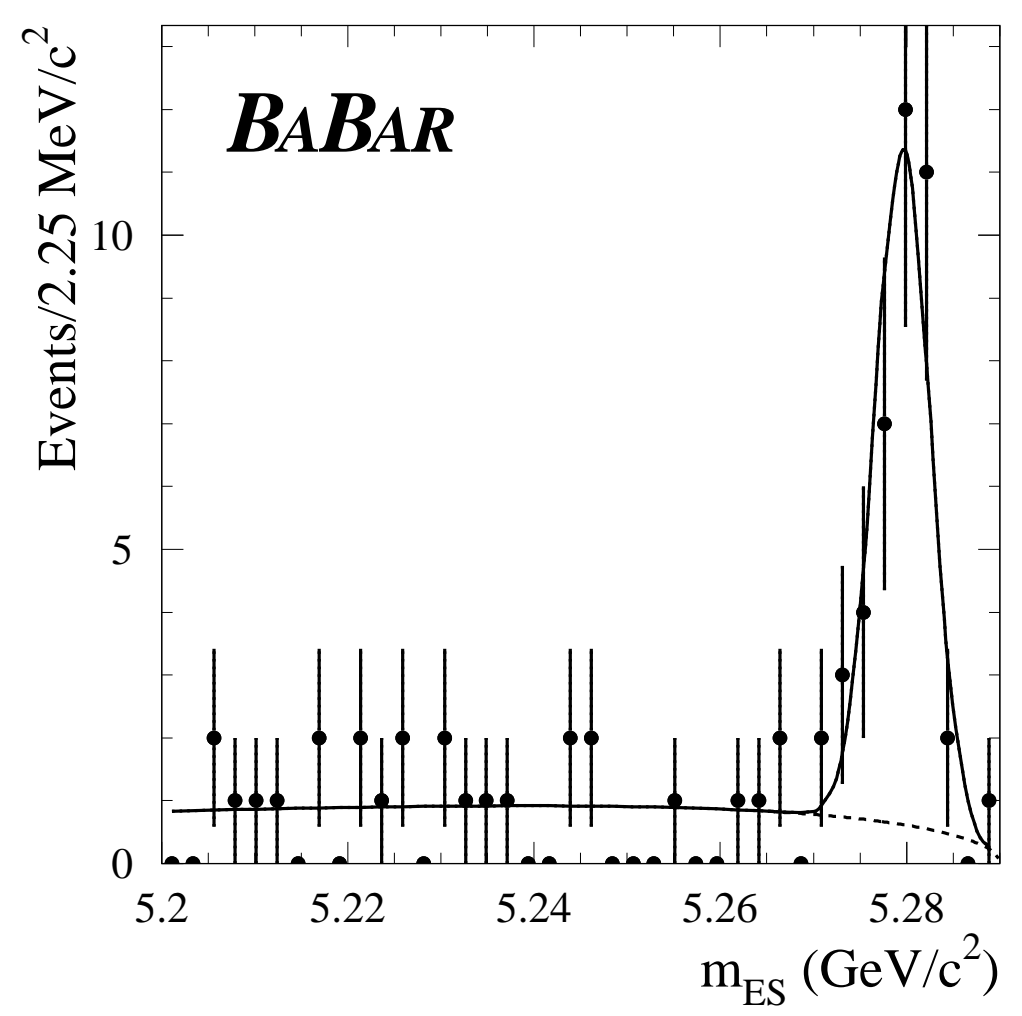

Figure 4: Distribution of events in $m_{\mathrm{ES}}$ plane with a cut of $|\Delta E|<25 \mathrm{MeV}$ applied. The curve represents a fit to the distribution of the sum of a Gaussian to model the signal and an ARGUS function to model the background shape.

The dominant systematic uncertainty in this measurement comes from our level of understanding of the charged particle tracking efficiency (9.4\%). The high charged particle multiplicity in this decay mode makes this measurement particularly sensitive to tracking efficiency. Uncertainties were assigned on a per track basis for $\pi, K$ and slow $\pi$, and were added linearly due to large correlations. The imprecisely known partial-wave content of the $B^{0} \rightarrow D^{*+} D^{*-}$ final state is another source of systematic uncertainty $(6.6 \%)$. This was estimated by calculating the change in the reconstruction efficiency for different final angular states in Monte Carlo. Other significant systematic uncertainties arise due to the uncertainties on the $D^{*+}, D^{0}$ and $D^{+}$branching fractions $(5.6 \%)$ and the differences in mass resolutions between Monte Carlo and data (4.1\%). The total systematic uncertainty from all sources is $14.5 \%$.

\section{$3 \quad$ Summary}

Using about $23 \mathrm{M} B \bar{B}$ events, we have observed several hundred completely reconstructed $B \rightarrow$ $D^{(*)} \bar{D}^{(*)} K$ decays. The following preliminary branching fractions have been measured: $\mathcal{B}\left(B^{0} \rightarrow\right.$ $\left.D^{*-} D^{0} K^{+}\right)=(2.8 \pm 0.7 \pm 0.5) \times 10^{-3}$ and $\mathcal{B}\left(B^{0} \rightarrow D^{*-} D^{* 0} K^{+}\right)=(6.8 \pm 1.7 \pm 1.7) \times 10^{-3}$ in good agreement with the CLEO measurements $\mathcal{B}\left(B^{0} \rightarrow D^{*-} D^{0} K^{+}\right)=\left(4.5_{-1.9}^{+2.5} \pm 0.8\right) \times 10^{-3}$ and $\mathcal{B}\left(B^{0} \rightarrow D^{*-} D^{* 0} K^{+}\right)=\left(13.0_{-5.8}^{+7.8} \pm 3.6\right) \times 10^{-3}$ [14]. We have observed an excess of $8.2 \pm 3.5$ events over a background of 1.7 events in the color-suppressed decay mode $B^{+} \rightarrow D^{*+} D^{*-} K^{+}$, 
where no significant number of candidates has been previously seen. The corresponding preliminary branching fraction is measured to be $\mathcal{B}\left(B^{+} \rightarrow D^{*-} D^{*+} K^{+}\right)=(3.4 \pm 1.6 \pm 0.9) \times 10^{-3}$. Finally, several candidates have also been observed in the $C P$ eigenstate $B^{0} \rightarrow D^{(*)+} D^{(*)-} K_{S}^{0}$. This study confirms that the transitions $b \rightarrow c \bar{c} s$ can proceed through the decays $B \rightarrow D^{(*)} \bar{D}^{(*)} K$. We have observed a signal of $31.8 \pm 6.2 \pm 0.4$ events in the decay $B^{0} \rightarrow D^{*+} D^{*-}$. We measure a preliminary branching ratio to be $\mathcal{B}\left(B^{0} \rightarrow D^{*+} D^{*-}\right)=(8.0 \pm 1.6 \pm 1.2) \times 10^{-4}$ in good agreement with the CLEO measurements $\mathcal{B}\left(B^{0} \rightarrow D^{*+} D^{*-}\right)=\left(9.9_{-3.3}^{+4.2} \pm 1.2\right) \times 10^{-4}$ 15.

\section{References}

[1] ARGUS Collaboration, H. Albrecht et al., Z. Phys. C54, 1 (1992).

[2] CLEO Collaboration, D. Gibaut et al., Phys. Rev. D53, 4734 (1996).

[3] CLEO Collaboration, S. Ahmed et al., Phys. Rev. D62, 112003 (2000).

[4] BABAR Collaboration, B. Aubert et al., BABAR-CONF-01/27, hep-ex/0107060.

[5] T. Browder, Hadronic decays and lifetimes of B and D mesons, Proceedings of the 1996 Warsaw ICHEP conference, Z. Ajduk and A.K. Wroblewski Eds, World Scientific (1997) p1139.

[6] I. I. Bigi, B. Blok, M. Shifman and A. Vainshtein, Phys. Lett. B323, 408 (1994).

[7] G. Buchalla, I. Dunietz and H. Yamamoto, Phys. Lett. B364, 188 (1995).

[8] I. Dunietz et al., Phys. Rev. D43, 2193 (1991).

[9] BABAR Collaboration, B. Aubert et al., SLAC-PUB-8596, hep-ex-0105044, to be published in Nucl.Inst.Methods.

[10] ARGUS Collaboration, H. Albrecht et al., Phys. Lett. B185, 218 (1987).

[11] "GEANT, Detector Description and Simulation Tool", CERN program library longwritup W5013(1994).

[12] Particle Data Group, D.E. Groom et al., Eur. Phys. J. C15, 1 (2000).

[13] T. Browder et al., Phys. Rev D61, 054009 (2000).

[14] CLEO Collaboration, CLEO CONF 97-26, EPS97 337.

[15] E. Lipeles et al., Phys. Rev. D62, 032005 (2000). 